\title{
Early Lung Cancer Detection Using Deep Learning Optimization
}

\author{
https://doi.org/10.3991/ijoe.v16i06.13657
Ahmed Elnakib (®), Hanan M. Amer
Mansoura University, Mansoura, Egypt
nakibemans.edu.eg \\ Fatma E.Z. Abou-Chadi \\ The British University, Cairo, Egypt
}

\begin{abstract}
This paper proposes a computer aided detection (CADe) system for the early detection of lung nodules from low dose computed tomography (LDCT) images. The proposed system initially preprocesses the raw data to improve the contrast of the low dose images. Compact deep learning features are then extracted by investigating different deep learning architectures, including Alex, VGG16, and VGG19 networks. To optimize the extracted set of features, a genetic algorithm (GA) is trained to select the most relevant features for early detection. Finally, different types of classifiers are tested in order to accurately detect the lung nodules. The system is tested on 320 LDCT images from 50 different subjects, using an online public lung database, i.e., the International Early Lung Cancer Action Project, I-ELCAP. The proposed system, using VGG19 architecture and SVM classifier, achieves the best detection accuracy of $96.25 \%$, sensitivity of $97.5 \%$, and specificity of $95 \%$. Compared to other state-of-the-art methods, the proposed system shows promising results.
\end{abstract}

Keywords - Lung nodule detection, lung cancer, early detection, deep learning

\section{Introduction}

Worldwide, cancer is the second leading cause of death after cardiovascular diseases. The American Cancer Society (ACS) estimated a number of 1,762,450 new cancer cases in 2019, accounting for 606,880 deaths in the United States [1]. 13\% of all these new cases are lung cancer (228,150 new cases), accounting for 142,670 deaths [1]. This number is about $24 \%$ of all cancer deaths, which is the highest mortality rate among all type of cancers [1]. Therefore, there is an urgent need to investigate how to improve the survival rate of lung cancer.

A recent study in the journal of nature medicine [2] reported that using low dose computed tomography (LDCT) can reduce the mortality rate by more than $20 \%$, therefor it has been recommended in the typical United State screening. The main advantage of using LDCT screening is its ability to show high resolution chest images with safer $\mathrm{x}$-ray dosage. Manual analysis of CT images to detect lung nodules is time consuming 
and subjective. In addition, it suffers from inter- and intra-subject variabilities. Therefore, several research teams have developed computer aided detection (CADe) systems in order to aid the radiologist to accurately detect nodules [3-5]. One of the main challenges in CADe system design is the detection of early cancerous nodules. At early lung

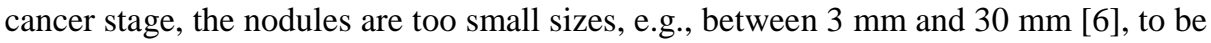
detected. However, clinical reports show that early detection is a major key to significantly improve the survival rate of lung cancer [7].

This study aims at developing a CADe system for early lung cancer detection. To do this job, data from the International-Early Lung Cancer Action Project, I-ELCAP [8], are collected.

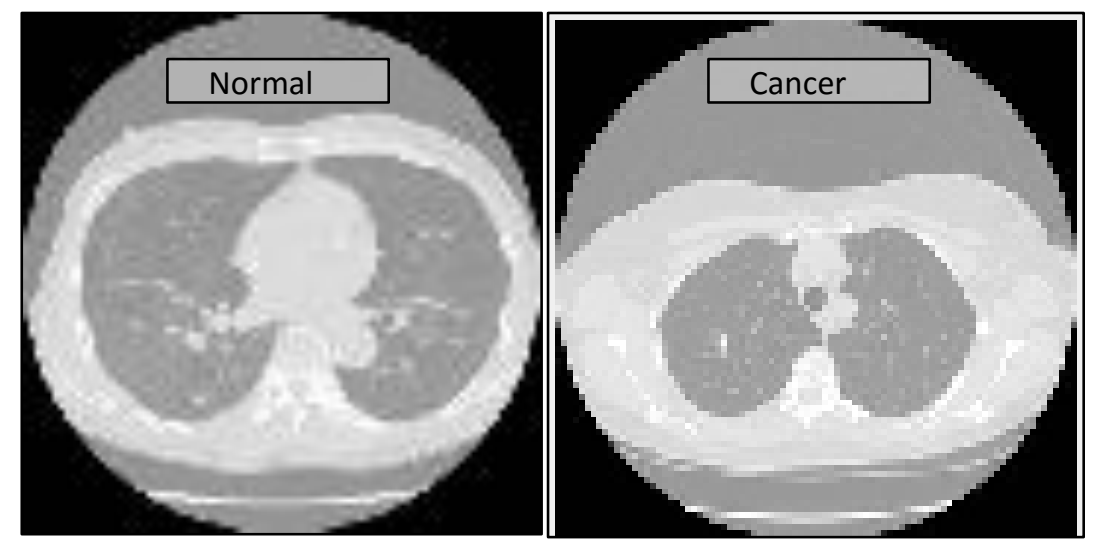

Fig. 1. Typical normal and cancerous LDCT image samples acquired from I-ELCAP

The proposed CADe system is composed from four steps (see Figure. 1):

i. Initially preprocess the raw LDCT data in order to improve its contrast,

ii. Extract compact deep learning features from the LDCT image,

iii. Optimize the extracted features in order to improve the detection accuracy,

iv. Classify the LDCT as normal or cancerous, based on the optimized feature vector. The main contributions of the proposed CADe system are two-folds:

- Analysis of extracting different deep learning features from different architectures, including Alex [9] and VGG16 and VGG19 [10] networks

- Optimization of the extracted deep learning features, using a smart genetic algorithm, running during the training phase of the CADe system, which does not only improve the accuracy of detection, but also reduces the feature dimensions, making the classification faster. 


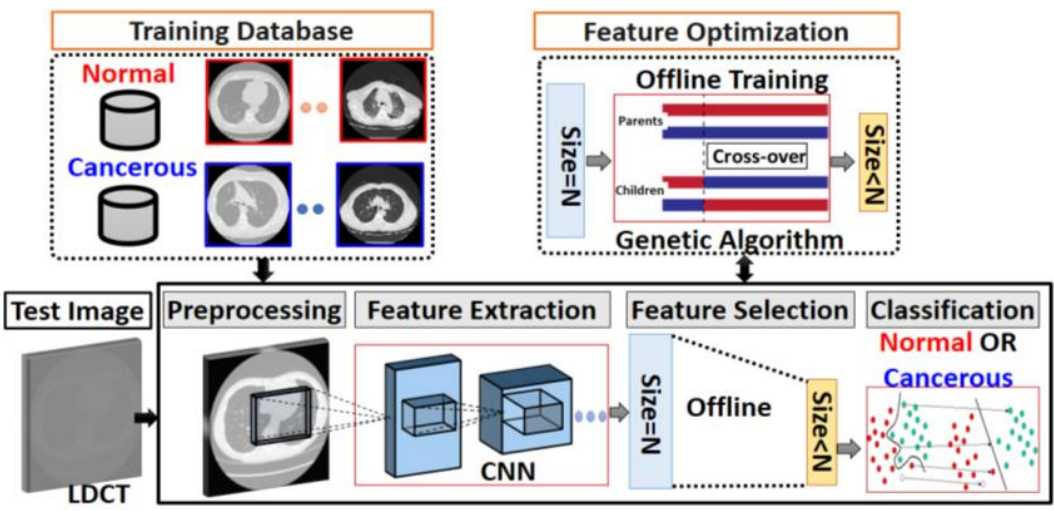

Fig. 2. Proposed CADe system for nodule detection composed of four steps: Preprocessing, feature extraction using $\mathrm{CNN}$, feature selection using offline genetic algorithm training, and classification, the output is either normal or cancerous

The current paper is an extension of our published paper in [11], with the following contributions:

i. Investigating more architectures rather than Alex network, including VGG16 and VGG19,

ii. Investigating more classifiers rather than the supported vector machine (SVM) used in [11], including K-nearest neighbor $(\mathrm{KNN})$, and decision trees, and

iii. Adding more experimentations and discussions in order to precisely quantify the advantages and limitations of the proposed CADe system.

The rest of the paper is organized as following. Section 2 outlines the related work for lung nodule detection. Section 3 details the proposed CADe system. Section 4 demonstrates the experimental results and related discussions. Finally, Section 5 concludes the paper and outlines the future work.

\section{$2 \quad$ Literature Review}

In the literature, several research groups have developed different CADe systems for cancerous lung nodule detection. These systems can be categorized as traditional and deep Convolutional Neural Network (CNN) systems. In this section, each category will be overviewed, as well as its strength and limitations.

\subsection{Traditional CADe systems}

Traditional CADe systems involve four main steps: lung segmentation, feature extraction, nodule detection, and false positive reduction [5]. These methods usually extract different features from the segmented lung regions in order to differentiate between normal and cancerous lung images. The extracted features may include intensitybased features, statistical features, textures, and/or nodule shapes. For example, Setio 
et al. [12] applied a 3D lung segmentation technique to identify the lung regions from the CT images. A feature vector, composed of 24 intensity-based, blobness, shape, and texture features were extracted from the segmented lung regions in order to detect lung nodules. A radial basis SVM was further used for the detection of large nodules. Li et al. [13] segmented the lung regions using a threshold-based technique. A set of intensity-based and texture features, including contrast, energy, correlation, homogeneity, and grey level concurrence matrix (GLCM), were extracted from the segmented lungs. Then a SVM was used for classification. Amer et al. [14] segmented the lung regions using bi-thresholding and morphological operations. A set of statistical, texture, histogram-based, wavelet features were fused using a genetic algorithm, to perform early lung nodule detection. Although traditional CADe system has achieved a considerable success in the detection of lung nodules, more sophisticated features are still need to be investigated in order to afford more accurate detection, especially for early cases.

\subsection{Deep CNN CADe systems}

Recently, deep learning CNNs have shown a remarkable success for lung nodule detection [3-4]. In CNN, medical images are directly processed, most often without segmenting the lung fields, throughout several convolutional layers that work as spatially localized filters and fully connected layers. These CNN architectures involve a very large number of parameters or weights in order to encode the images into a compact high-level feature space. To adjust the CNN parameters, training should be performed using a very large number of data images to avoid under-fitting. The extracted deep learned feature vector has shown a significant capability to describe precisely the training data and distinguish between normal and cancerous lung nodules [15]. For example, Jin et al. [16] trained a 3D CNN architecture, composed of eleven layers, using the segmented lung regions for the task of lung nodule detection. Their method achieved a detection accuracy of $87.5 \%$. Shen et al. [17] achieved a detection accuracy of $87.14 \%$ using a CNN architecture that involved a multi-crop pooling of convolutional layers. Wang et al. [18] applied a multi-level feature pyramid network followed by a $3 \mathrm{D}$ non-maximum suppression and a $3 \mathrm{D} \mathrm{CNN}$ to achieve a sensitivity of $95.8 \%$ over the LUng Nodule Analysis (LUNA16) dataset [18]. More recently, Winkels et al. [19] showed that using 3D CNN with group convolutions was able to reduce the number of false positives for lung nodules detection. The promising results of using deep learning CADe systems throughout the literature encourage us to adopt using this approach to extract the LDCT image features.

\section{Methods}

The proposed CADe system includes four processing stages, illustrated in Figure 1. Firstly, the test LDCT image is input to a preprocessing stage, where contrast of image is improved. Secondly, a CNN model is used to extract a compact feature vector that describes the LDCT images. Thirdly, a feature selection stage is applied to select the most relevant features for the task of pulmonary nodule detection. The selected set of 
relevant features are determined offline using a smart genetic algorithm (GA), which significantly reduces the feature space dimensions and improves the detection accuracy. Finally, a classification stage is applied to determine whether the test LDCT image contains pulmonary nodules or not. In this section, we will detail the procedures of each of these stages.

\subsection{Database description}

The LDCT images are collected from an online publically available database, i.e., the Early Lung Cancer Action Project (ELCAP) database [8]. In order to test the proposed CADe system, 320 LDCT images from 40 different subjects are selected randomly, including 160 normal cross-sections, and 160 cancerous ones, to avoid the bias during experimentations. In order to account for early lung nodule detection, nodules are in the range between $3 \mathrm{~mm}$ to $30 \mathrm{~mm}$. Images are of resolution of $0.76 \times 0.76 \times 1.25$ $\mathrm{mm}$. Typical normal and cancerous samples of LDCT images, from the I-ELCAP project, are exemplified in Figure. 2.

\subsection{Preprocessing of LDCT images}

Preprocessing stage is implemented in three steps, as shown in Figure 3. First, the contrast of the raw image is enhanced using the histogram stretching technique [19]. Second, a smoothing Wiener filter is applied in order to remove the scanner noise. Finally, the image is cropped to the standard size of the CNN model that is used for feature extraction (i.e., $227 \times 227$ for Alex architecture, and $224 \times 224$ for VGG16 and VGG19 architectures, see Table. 1).

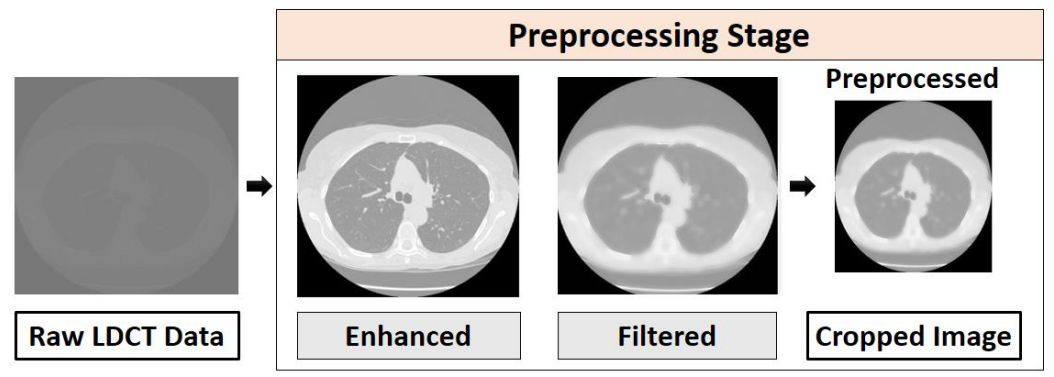

Fig. 3. Preprocessing raw LDCT data in three steps: histogram stretching to improve image contrast, image smoothing using Wiener filer, and cropping to the standard input size of the CNN model used for feature extraction

\subsection{Feature extraction using $(\mathrm{CNN})$}

To extract useful features that can describe the LDCT images, three popular CNN architectures are investigated, namely, Alex [9], VGG16, and VGG19 networks [10]. A detailed comparison between these architectures is provided in Table 1 [21]. To ovoid 
under-fitting, these architectures were originally trained over a large number of images (1.2 million for Alex network [9], and 1.3 Million for VGG16 and VGG19 [10], in order to adjust their huge number of parameters ( 60, 138, and 144 million for Alex, VGG16, and VGG19, respectively). In our experiments, we investigate using these different CNN models to provide a reliable descriptor for LDCT images. Since the collected data size is small (320 LDCT images), the proposed CADe system applies transfer learning, where the parameters of the convolutional layers are kept unchanged (trained using the original data in $[9,10]$ ) and only the fully connected (FC) layers are trained and customized using the new database of interest. Transfer leaning is repeatedly applied by different research teams, showing a remarkable success [22]. To apply the transfer learning, the last fully connected (FC) layer (output layer) is customized based on the number of classes, i.e., two neurons in our experiments (normal or cancerous). In the proposed CADe system, the FC layer, just before the output layer, is used as a compact high-level feature descriptor (of length 4096 for all the three investigated models, as shown in Table 1 to precisely describe the LDCT image.

Table 1. Comparison between the different CNN models that are used for feature extraction (Alex, VGG16, and VGG19) with respect to the input space dimention, the number of layers (convolutional (conv), fully connected (FC), and total number of layers $\left(\sum\right)$ ), the number of the training images originally used, in millions (M), the number of network parameters, in milions $(\mathrm{M})$, and the extracted feature size used in our experiments (i.e., the size of the FC layer just before the output layer)

\begin{tabular}{|c|c|c|c|c|c|c|c|}
\hline \multirow{2}{*}{ Model } & \multirow{2}{*}{ Input size } & \multicolumn{3}{|c|}{ \# layers } & \multirow{2}{*}{$\begin{array}{l}\text { \# Training } \\
\text { (M) }\end{array}$} & \multirow{2}{*}{$\begin{array}{c}\text { \# Parameters } \\
\text { (M) }\end{array}$} & \multirow{2}{*}{ Feature size } \\
\hline & & \#conv & $\# F C$ & $\sum$ & & & \\
\hline Alex & $227 \times 227$ & 5 & 3 & 8 & 1.2 & 60 & 4096 \\
\hline VGG 16 & $224 \times 224$ & 13 & 3 & 16 & 1.3 & 138 & 4096 \\
\hline VGG 19 & $224 \times 224$ & 16 & 3 & 19 & 1.3 & 144 & 4096 \\
\hline
\end{tabular}

\subsection{Genetic Algorithm (GA) feature optimization}

Since transfer learning keeps the convolutional layers' parameters unchanged (are not trained with the database of interest), the extracted feature vector is generic and may involve redundant information. In order to select the most relevant features for lung nodule detection, a smart GA is designed. The output of the GA is a binary chromosome of length 4096 bits, evaluated offline at the training phase of the CADe system, in which a bit of logic ' 1 ' indicates that this feature is relevant and a bit of logic ' 0 ' indicates that this feature is irrelevant, so it is removed from the optimized feature vector used in the test phase. The detailed of GA design is illustrated in Figure. 4.

As shown in Figure 4, the parameters of the GA are set (e.g., population size, number of generations, mutation probability, etc.). Then, the GA initially generates a random population of binary chromosomes, each of the dimensions of size 4096, i.e., the size of the extracted feature vector from the CNN model. The fitness function is then evaluated for each chromosome in the population. This fitness function is defined as the accuracy of detection (AD) [23]: 


$$
A D=\frac{T P+T N}{T P+T N+F P+F N}
$$

Where $T P, T N, F P$, and $F N$ denotes the true positive, true negative, false positive, and false negative, respectively.

For feature selection, a subset of training images is iteratively processed by the GA (i.e., one half of the training images and the other half is used to train the classifier; the last step in the proposed CADe system). At each iteration, genetic operations (selection, cross over, and mutation) are applied in order to form a new population. The process is repeated until the detection accuracy (fitness, $A D$ ) no longer improves. After GA termination, the chromosome of the best fitness (maximum detection accuracy, $A D$ ) represents the optimal feature vector that should be used for nodule detection, where a bit of ' 1 ' in the chromosome means that the feature is selected and a bit of ' 0 ' means that the feature is not selected (see Figure 4). The proposed CADe system uses the following GA setting: tournament selection [24], with a tournament size of four, blending crossover [25], and adaptive feasible mutation [26]. The number of generations is set to a maximum of 100 generations.

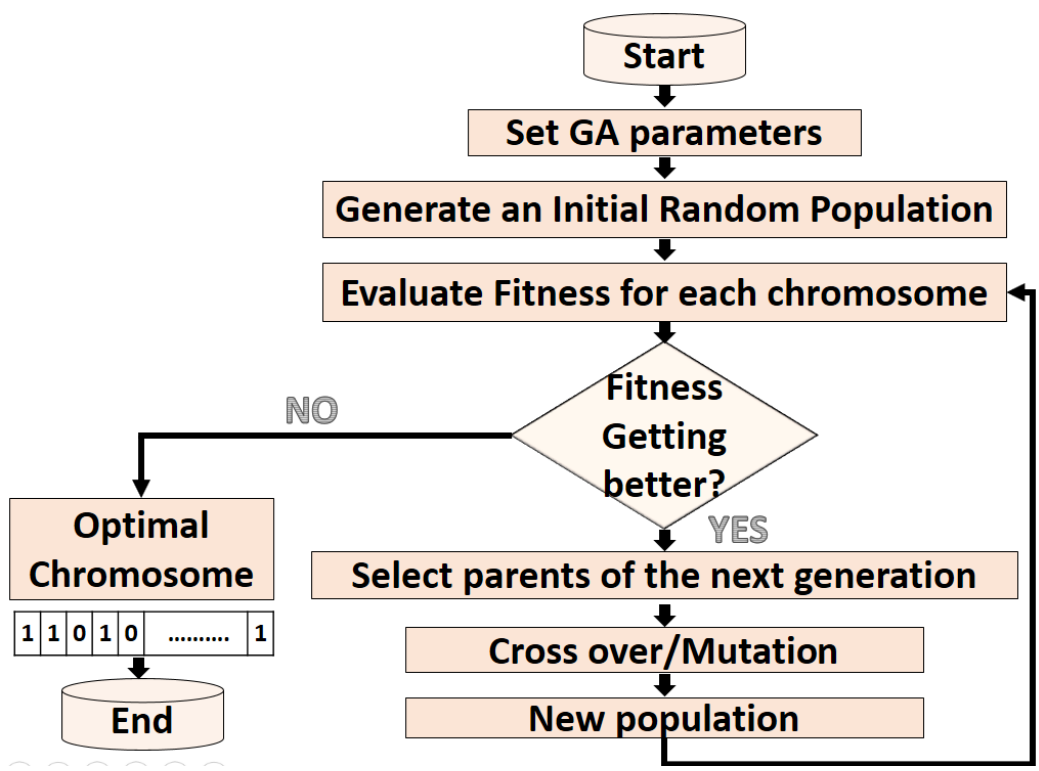

Fig. 4. Details of the Genetic Algorithm (GA) proposed for feature selection at the training phase. Binary chromosomes are selected, where a bit of ' 1 ' in the chromosome means that the feature is selected and a bit of ' 0 ' means the feature is not selected

\subsection{Classification}

The last step in the proposed CADe system is to classify the LDCT images into normal or cancerous, based on the reduced-dimension feature descriptor, optimized using the GA, trained using one half of the training images. The rest of the training images 
(the other half) is used to train the classifier. For this job, we investigate different number of classifiers, including, K-Nearest Neighbor (KNN), decision trees, and supported vector machines (SVM) with binary linear kernel in order to detect the pulmonary lung nodules.

\subsection{Performance metrics}

To evaluate the efficiency of the proposed CADe system, standard detection metrics are used on the test data, namely, the accuracy of detection $(A D)$ (see Equation (1)), the specificity $(S p)$, and the sensitivity $(S)$, defined as follows [23]:

$$
\begin{gathered}
S P=\frac{T N}{T N+F P} \\
S=\frac{T P}{T P+F N}
\end{gathered}
$$

\section{Experimental Results and Discussion}

Collected data (320 image) is divided into $75 \%$ training and $25 \%$ test (see Table. 2 ). Training is carried throughout two phases, each with equal number of training images. The first phase is GA training, in order to select the most relevant features for the task of pulmonary nodule detection. The second phase is classifier training, based on the selected feature vector, in order to improve the detection accuracy. The details of the train and test datasets are illustrated in Table. 2. Note that all experiments select equal number of normal and cancerous images to avoid any bias.

Table 2. Data is divided into $75 \%$ for training and $25 \%$ for test. Training is performed in two phases. Training the GA to select the most releavant features and training the classifier based on the selected relevant features. Normal and cancerous images are selected

\begin{tabular}{|c|c|c|c|c|c|}
\hline & \multicolumn{3}{|c|}{ Train } & \multirow{2}{*}{ Test } & \multirow{2}{*}{ Total } \\
\hline & Phase I GA & Phase II Classifier & Total & & \\
\hline Percentage & $37.5 \%$ & $37.5 \%$ & $75.0 \%$ & $25.0 \%$ & $100 \%$ \\
\hline \# Normal & 60 & 60 & 120 & 40 & 160 \\
\hline \# Cancerous & 60 & 60 & 120 & 40 & 160 \\
\hline \# Total & \multicolumn{3}{|c|}{240} & 80 & 320 \\
\hline
\end{tabular}
equally for each experiment to avoid the bias

In order to investigate the potential of the different CNN models to extract useful features that can describe the LDCT images, we test using all the feature vector of length 4096 of the last FC layer, just before the output layer of each model, for the task of pulmonary nodule detection. Three different types of classifiers are investigated in order to obtain the best detection accuracy, namely, KNN, decision trees, and SVM. As reported results in Table. 3, using SVM achieves the best detection accuracies, $A D$, of $88.8 \%, 91.2 \%$, and $86.3 \%$ using Alex, VGG16, and VGG19 models, respectively. These results highlight that these CNN models can extract useful features that can be 
efficiently used for nodule detection. In addition, these results indicate the superior of using SVM over other classifiers due to its sophisticated nature.

To further optimize the feature descriptor, the proposed GA optimization is investigated, using the best type of classifier (SVM). As shown in Table. 3, the GA step can improve the detection accuracies to $92.5 \%$ ([11]), 91.3\%, 96.3\% using Alex, VGG16, and VGG19, respectively. In addition, the GA optimization was able to reduce the feature dimensions from 4096 to 1822, 1925, and 1925, for Alex, VGG16, and VGG19, respectively. These results show the promise of using GA in the proposed CADe system to improve the overall system detection accuracy and to speed up the classifier, removing the burden of irrelevant features. Figure 5 shows visual samples of the correctly classified LDCT images from different subjects using the proposed CADe system with GA optimization and SVM classifier using the three different CNN models.

Table 3. Experimental results of the proposed CADe system for early detection interms of three metrics $(A D, S, S p)$, with differnt architectures (Alex, VGG16, and VGG19) and different settings: whether the proposed GA is used (YES) or not (NO), the classifer feature size, and the type of classifier used (KNN, decision tree (TREE), or SVM)

\begin{tabular}{|l|l|l|l|l|l|l|}
\hline CNN Model & \multicolumn{1}{|c|}{ GA } & Feature size & Classifier & \multicolumn{1}{c|}{$\boldsymbol{A D}$} & \multicolumn{1}{c|}{$\boldsymbol{S}$} & \multicolumn{1}{c|}{$\boldsymbol{S p}$} \\
\hline \multirow{4}{*}{ ALEX } & NO & 4096 & KNN & $81.3 \%$ & $85.0 \%$ & $97.5 \%$ \\
\cline { 2 - 7 } & NO & 4096 & TREE & $83.75 \%$ & $92.5 \%$ & $92.5 \%$ \\
\cline { 2 - 7 } & NO & 4096 & SVM & $88.8 \%$ & $75.0 \%$ & $97.5 \%$ \\
\hline \multirow{5}{*}{ VLEX [11] } & YES & 1822 & SVM & $92.5 \%$ & $90 \%$ & $95 \%$ \\
\hline \multirow{5}{*}{ VGG16 } & NO & 4096 & KNN & $87.5 \%$ & $80 \%$ & $95 \%$ \\
\cline { 2 - 7 } & NO & 4096 & TREE & $87.5 \%$ & $80 \%$ & $95 \%$ \\
\cline { 2 - 7 } & NO & 4096 & SVM & $91.2 \%$ & $87.5 \%$ & $95 \%$ \\
\cline { 2 - 7 } & NOS & 1925 & SVM & $91.3 \%$ & $90 \%$ & $92.5 \%$ \\
\cline { 2 - 7 } & NO & 4096 & KNN & $82.5 \%$ & $67.5 \%$ & $97.5 \%$ \\
\cline { 2 - 7 } & NO & 4096 & TREE & $82.5 \%$ & $67.5 \%$ & $97.5 \%$ \\
\hline \multirow{2}{*}{ VGG19 (Best) } & YES & 4096 & SVM & $86.3 \%$ & $80.3 \%$ & $92.5 \%$ \\
\hline
\end{tabular}




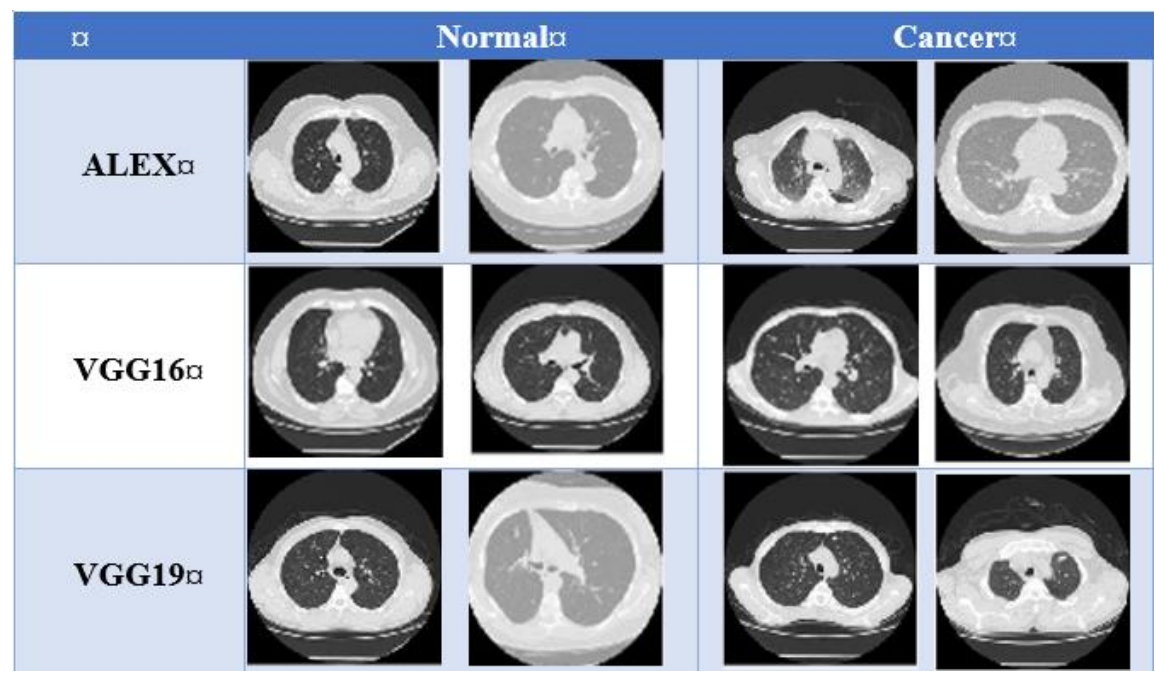

Fig. 5. Samples of correctly classified normal (first two columns) and cancerous (last two columns) images using the proposed CADe system, with Alex (first row), VGG16 (second row), and VGG 19 (third row) models

To discuss the limitations of the proposed CADe system, Figure 6 exemplified samples of false positives and false negatives of our system. As shown in samples, the vascular structure is close to nodule shapes, leading to false positive and false negative cases. In the future, we will investigate the fusion of the different model features in order to improve the accuracy.

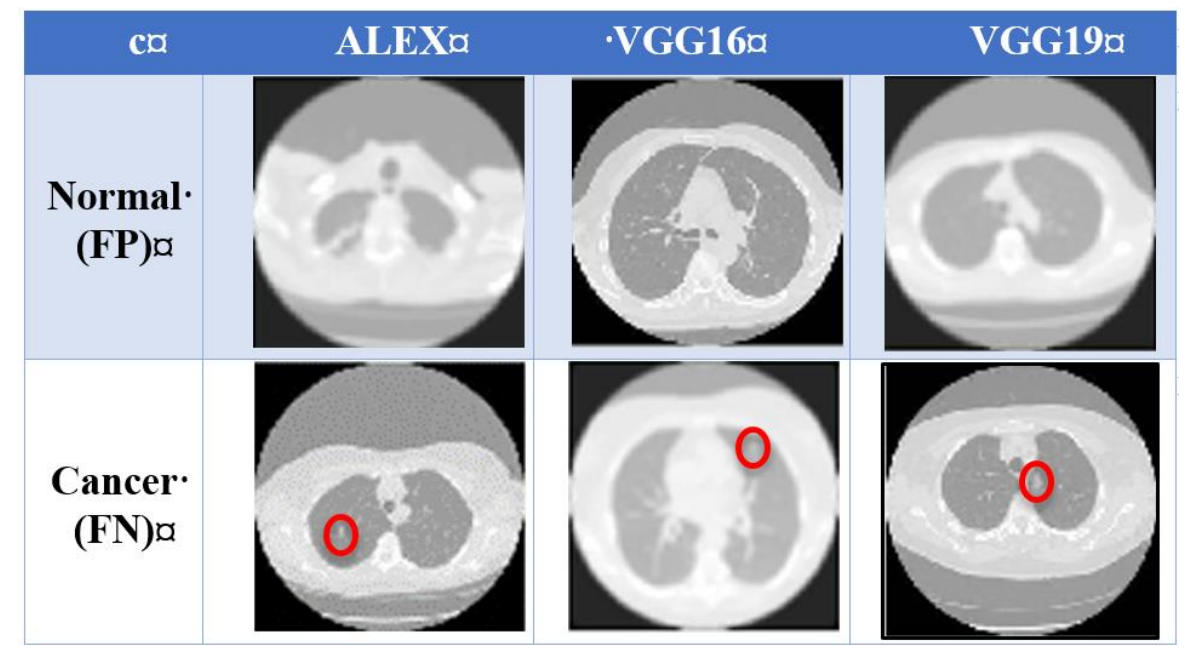

Fig. 6. Samples of false positives (misclassified normal images, first row) and false negatives (misclassified cancer images, second row) of the proposed CADe system using GA and SVM classier. Red circles indicate the cancerous nodules 


\section{Conclusion}

A CADe system for early pulmonary nodules is presented. The CADe system is based on transfer learning to get a generic feature descriptor and a smart genetic algorithm (GA) to select the most relevant features for early pulmonary cancer detection. The CADe system accuracy is tested on an online publically available database; the Early Lung Cancer Action Project (ELCAP). The proposed CADe system shows promising results in early lung cancer detection with respect to the state-of-the-art methods. In the future, more databases will be investigated in order to stand on the robustness of the proposed system.

\section{References}

[1] Siegel, R. L., Miller, K. D., and Jemal, A. (2019). Cancer statistics, 2019. CA: a cancer journal for clinicians, 69(1), 7-34. https://doi.org/10.3322/caac.21551

[2] Ardila, D., Kiraly, A. P., Bharadwaj, S., Choi, B., Reicher, J. J., Peng, L, Tse, D., Etemadi, M., Ye, W., Corrado, G. and Naidich, D. P. (2019). End-to-end lung cancer screening with three-dimensional deep learning on low-dose chest computed tomography. Nature medicine, 25(6), 954. https://doi.org/10.1038/s41591-019-0447-X

[3] El-Regaily, S. A., Salem, M. A., Abdel Aziz, M. H., and Roushdy, M. I. (2018). Survey of computer aided detection systems for lung cancer in computed tomography. Current Medical Imaging Reviews, 14(1):3-18. https://doi.org/10.2174/1573405613666170602123329

[4] Zhang, G., Jiang, S., Yang, Z., Gong, L., Ma, X., Zhou, Z., Bao, C. and Liu, Q. (2018). Automatic nodule detection for lung cancer in CT images: A review. Computers in biology and medicine, 103:287-300. https://doi.org/10.1016/j.compbiomed.2018.10.033

[5] El-Baz, A., Beache, G. M., Gimel'farb, G., Suzuki, K., Okada, K., Elnakib, A., Soliman, A., and Abdollahi, B. (2013). Computer-aided diagnosis systems for lung cancer: challenges and methodologies. International journal of biomedical imaging, 2013. https://doi.org/10.1155/2013/942353

[6] Jacobs, C., and van Ginneken, B. (2019). Google's lung cancer AI: a promising tool that needs further validation. Nature Reviews Clinical Oncology, 1. https://doi.org/10.1038 /s41571-019-0248-7

[7] Pompe, E., de Jong, P. A., and Hoesein, F. A. M. (2019). Unravelling complexities of the subsolid pulmonary nodule-detection, characterization, natural history, monitoring and (future) patient management. Journal of Thoracic Disease, 11(Suppl 9):S1402. https://doi.org/10.21037/jtd.2019.03.07

[8] Early Lung Cancer Action Program (ELCAP), available from: http://www.via. cornell.edu/lungdb.html

[9] Krizhevsky, A., Sutskever, I., \& Hinton, G. E. (2012). Imagenet classification with deep convolutional neural networks. Proceedings of the Advances in neural information processing systems. pp. 1097-1105

[10] Simonyan, K., \& Zisserman, A. (2014). Very deep convolutional networks for large-scale image recognition. arXiv preprint arXiv: 1409.1556.

[11] Elnakib, A., Amer, H. M., and Abou-Chadi, F. E. (2019, April). Computer aided detection system for early cancerous pulmonary nodules by optimizing deep learning features. In Proceedings of the 2019 8th International Conference on Software and Information Engineering (pp. 75-79). ACM. https://doi.org/10.1145/3328833.3328856 
[12] Setio, A. A., Jacobs, C., Gelderblom, J., and van Ginneken, B. (2015). Automatic detection of large pulmonary solid nodules in thoracic CT images. Medical physics, 42(10): 56425653. https://doi.org/10.1118/1.4929562

[13] Li, L., Wu, Y., Yang, Y., Li, L., and Wu, B. (2018, June). A New Strategy to Detect Lung Cancer on CT Images. 2018 IEEE 3rd International Conference on Image, Vision and Computing (ICIVC). pp 716-722 https://doi.org/10.1109/ICIVC.2018.8492820

[14] Amer, H. M., Abou-Chadi, F. E., Kishk, S. S., and Obayya, M. I. (2018, May). A Computer-Aided Early Detection System of Pulmonary Nodules in CT Scan Images. 7th International Conference on Software and Information Engineering. pp. 81-86. https://doi.org/10.11 $\underline{45 / 3220267.3220291}$

[15] Setio, A. A. A., Traverso, A., De Bel, T., Berens, M. S., van den Bogaard, C., Cerello, P., Chen, H., Dou, Q., Fantacci, M.E., Geurts, B. and van der Gugten, R. (2017). Validation, comparison, and combination of algorithms for automatic detection of pulmonary nodules in computed tomography images: the LUNA16 challenge. Medical image analysis, 42:1-13. https://doi.org/10.1016/j.media.2017.06.015

[16] Jin, T., Cui, H., Zeng, S., and Wang, X. (2017). Learning deep spatial lung features by 3D convolutional neural network for early cancer detection. International Conference on Digital Image Computing: Techniques and Applications (DICTA). pp. 1-6 https://doi.org/10.110 9/DICTA.2017.8227454

[17] Shen, W., Zhou, M., Yang, F., Yu, D., Dong, D., Yang, C., Zang, Y., and Tian, J. (2017). Multi-crop convolutional neural networks for lung nodule malignancy suspiciousness classification. Pattern Recognition, 61:663-673. https://doi.org/10.1016/j.patcog.2016.05.029

[18] Wang, B., Qi, G., Tang, S., Zhang, L., Deng, L., and Zhang, Y. (2018). Automated pulmonary nodule detection: High sensitivity with few candidates. In International Conference on Medical Image Computing and Computer-Assisted Intervention (pp. 759-767). Springer, Cham. https://doi.org/10.1007/978-3-030-00934-2 84

[19] Winkels, M., and Cohen, T. S. (2019). Pulmonary nodule detection in CT scans with equivariant CNNs. Medical image analysis, 55, 15-26. https://doi.org/10.1 016/j.media.2019.03.010

[20] Andrews, H. C. (1976). Monochrome digital image enhancement. Applied optics, 15(2):495-503. https://doi.org/10.1364/AO.15.000495

[21] Fu, J., and Rui, Y. (2017). Advances in deep learning approaches for image tagging. APSIPA Transactions on Signal and Information Processing, 6. https://doi.org/10.1 017/ATSIP.2017.12

[22] Litjens, G., Kooi, T., Bejnordi, B. E., Setio, A. A. A., Ciompi, F., Ghafoorian, M., Van Der Laak, J.A., Van Ginneken, B., and Sánchez, C. I. (2017). A survey on deep learning in medical image analysis. Medical image analysis, 42:60-88. https://doi.org/10.10 16/j.media.2017.07.005

[23] Yerushalmy, J. (1947). Statistical problems in assessing methods of medical diagnosis, with special reference to X-ray techniques. Public Health Reports (1896-1970): 1432-1449 https://doi.org/10.2307/4586294

[24] Miller, B. L., and Goldberg, D. E. (1995). Genetic algorithms, tournament selection, and the effects of noise. Complex systems, 9(3):193-212

[25] Eiben, A. E., and Schippers, C. A. (1998). On evolutionary exploration and exploitation. Fundamenta Informaticae, 35(1-4):35-50 https://doi.org/10.3233/FI-1998-35123403

[26] Thierens, D. (2002, May). Adaptive mutation rate control schemes in genetic algorithms. Proceedings of the 2002 Congress on Evolutionary Computation. CEC'02. pp. 980-985 


\section{$7 \quad$ Authors}

Ahmed Elnakib is an assistant professor, Electronics and Communications Engineering Department, Faculty of Engineering, Mansoura University, Mansoura, Egypt. He received his Ph.D. degree from university of Louisville, USA, in 2013. His current research includes developing computer aided diagnostic systems, machine learning, stochastic modeling, detection of brain disorders, and signal and image processing. $\mathrm{He}$ has authored or coauthored more than 50 technical articles, appeared in world-renown journals including IEEE TMI and Medical Physics. Mr. Elnakib is a regular reviewer for a number of technical journals including Neurocomputing, Medical Image Analysis, IEEE TMI and IEEE TIP. Email: nakib@mans.edu.eg

Hanan M. Amer is an assistant professor, Electronics and Communications Engineering Department, Faculty of Engineering, Mansoura University, Mansoura, Egypt. She received her Ph.D. degree from Mansoura University, Egypt, in 2018. Her field of experience includes signal and image processing, medical image analysis, and lung cancer. She has authored or coauthored more than 6 technical articles.

Fatma E. Z. Abou-Chadi is a professor and head of Electrical Engineering Department, Faculty of Engineering, The British University of Egypt, Cairo, Egypt. She received her BSc. degree from Ain-Shams University, Egypt, in 1974. In 1978, she received her Master degree in Electrical Communications Engineering from Mansoura University. She awarded the PhD degree at the Imperial College of Science and Technology, University of London, UK in 1986, specializing in Biomedical Signal Processing and Informatics. Prof. Abou-Chadi has authored and co-authored more than 90 scientific articles and two books. Her scientific interests and current research work include biomedical signal processing, medical imaging, digital signal processing, timeseries analysis, and information technology in biomedicine. Prof. Abou-Chadi is a Senior Member at Institute of Electrical and Electronics Engineers - IEEE since 1995, and a Member of the Society of IEEE Women in Engineering since 2000.

Article submitted 2020-02-09. Resubmitted 2020-03-25. Final acceptance 2020-03-29. Final version published as submitted by the authors. 